Discrete Comput Geom 29:511-524 (2003)

DOI: $10.1007 /$ s00454-003-2773-4

\title{
A General Notion of Visibility Graphs*
}

\author{
Mike Develin, ${ }^{1}$ Stephen Hartke, ${ }^{2}$ and David Petrie Moulton ${ }^{3}$ \\ ${ }^{1}$ Department of Mathematics, University of California at Berkeley, \\ Berkeley, CA 94720-3840, USA \\ develin@math.berkeley.edu \\ ${ }^{2}$ Department of Mathematics, Rutgers University, \\ Piscataway, NJ 08854, USA \\ hartke@math.rutgers.edu \\ ${ }^{3}$ Center for Communications Research, \\ 805 Bunn Dr., Princeton, NJ 08540, USA \\ moulton@idaccr.org
}

\begin{abstract}
We define a natural class of graphs by generalizing prior notions of visibility, allowing the representing regions and sightlines to be arbitrary. We consider mainly the case of compact connected representing regions, proving two results giving necessary properties of visibility graphs, and giving some examples of classes of graphs that can be so represented. Finally, we give some applications of the concept, and we provide potential avenues for future research in the area.
\end{abstract}

\section{Introduction}

At the AMS/MAA Joint Meetings in San Antonio in January of 1999, Alice Dean presented a paper on rectangle-visibility graphs. A graph is defined to be a rectanglevisibility graph if there exists a set of closed rectangles on $\mathbb{R}^{2}$, with sides parallel to the coordinate axes and disjoint except possibly for overlapping boundaries, such that each vertex corresponds to a rectangle and two vertices are joined by an edge if and only if there exists an unobstructed horizontal or vertical corridor of positive width connecting the two corresponding rectangles [4]. There are many other concepts of visibility graphs;

* The first two authors were financially supported by the University of Minnesota-Duluth and grants from the National Science Foundation (Grant DMS-92820179) and the National Security Agency (Grant 904-001-0026), and by NSF Graduate Research Fellowships. The third author was supported by the University of Wisconsin and the Center for Communications Research in Princeton. 
two of the most commonly used are the line-segment definition [8], [2] and the simple polygon definition [1], [9].

In this paper we consider visibility graphs corresponding to an extended notion of visibility. Namely, we define a sightline to be a line segment between points in two different regions intersecting no other region. A graph $G$ (all graphs we consider will be finite and simple) is then defined to be a visibility graph if there exists a set of disjoint nonempty connected regions in $\mathbb{R}^{2}$, with each region corresponding to a vertex of $G$, such that two vertices are connected by an edge in $G$ if and only if there exists a sightline between the corresponding regions. Throughout this paper we will identify vertices with the regions representing them.

Note that the regions are required to be connected. If we do not make this requirement, all connected graphs turn out to be visibility graphs by means of a simple construction. Let $G$ be an arbitrary connected graph; order the vertices $v_{1}, \ldots, v_{n}$ so that for all $1 \leq k \leq n$, the induced subgraph $\left.G\right|_{v_{1}, \ldots, v_{k}}$ is connected. Start with a disk representing $v_{1}$. For each $v_{i}$ in ascending order of $i$, cut a disk out of each area labeled $v_{j}$ for all $j<i$ such that $v_{j}$ and $v_{i}$ are adjacent in $G$. Label the interior of this disk $v_{i}$. It is then straightforward to check that this yields a visibility representation of $G$, as illustrated in Fig. 1.

By requiring the representing regions to be connected, we eliminate trivial constructions of this nature. In addition, we can require our representing regions to have other properties such as openness, compactness, and convexity; each of these yields a different theory of visibility graphs. We can restrict the theory further by choosing our regions from a severely limited list of objects, such as rectangles, disks, or even points. Naturally, with each restriction, the number of representable graphs decreases.

We are concerned primarily with compact visibility graphs, graphs that can be represented as visibility graphs using compact connected subsets of $\mathbb{R}^{2}$. (A subset $S \subset \mathbb{R}^{2}$ is compact if and only if it is closed and bounded; this is equivalent to requiring every infinite sequence $x_{1}, x_{2}, \ldots$ of points in $S$ to have a convergent subsequence.) This restriction allows us to give and prove a necessary property of graphs in this class, while
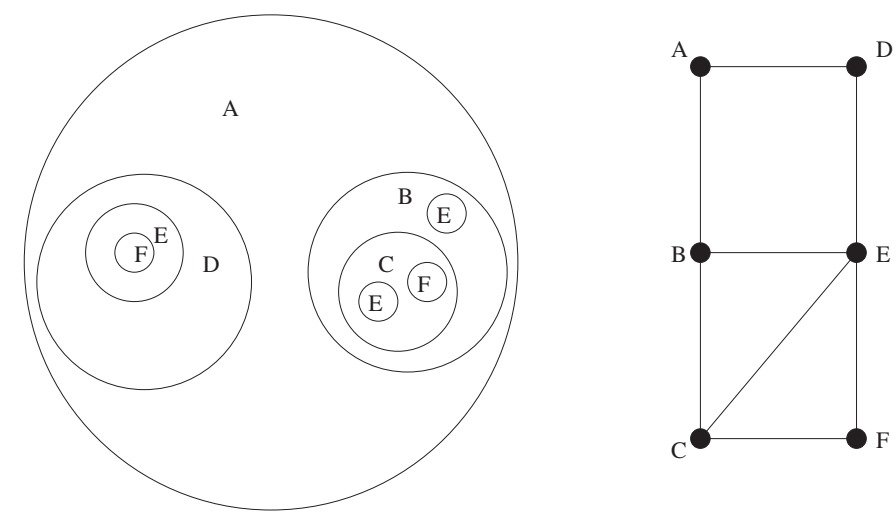

Fig. 1. A construction of an arbitrary connected graph as a visibility graph using disconnected regions. Each vertex is represented by the region consisting of the union of the areas bearing its letter. (This construction can be trivially modified to use compact regions.) 
at the same time preserving enough flexibility to facilitate visibility representations for a wide range of graphs.

\section{Convex Compact Visibility Graphs}

We begin by considering the case in which our representing regions are not only compact, but also convex. We define a graph to be a convex compact visibility graph, or if it can be realized as a visibility graph in $\mathbb{R}^{2}$ with all representing regions both compact and convex. One of our main theorems is the following, which gives a necessary condition on such graphs.

Theorem 1. If $G$ is a convex compact visibility graph, then every edge of $G$ must be either a bridge or part of a $K_{3}$.

The following lemma will be useful in the proof of the theorem.

Lemma 1. Suppose we have mutually visible regions $A$ and $B$. If any other region intersects a segment joining a point of $A$ and a point of $B$, then the edge $A B$ is part of $a$ $K_{3}$ in the corresponding visibility graph.

Proof. Take $a, a^{\prime} \in A$ and $b, b^{\prime} \in B$ so that $\overline{a b}$ is a sightline and $\overline{a^{\prime} b^{\prime}}$ intersects some other region. We have two cases: either $\overline{a b^{\prime}}$ is a sightline or it is not. It suffices to consider only the second case; if $\overline{a b^{\prime}}$ is a sightline, then switching the labels of the regions $A$ and $B$ and replacing $\left(a, b, b^{\prime}\right)$ by $\left(b^{\prime}, a, a^{\prime}\right)$ puts us in the situation of the second case.

Consider the straight line path $\varphi:[0,1] \rightarrow B$ from $b$ to $b^{\prime}$; as $B$ is convex, the image of this path lies entirely in $B$. Because all representing regions are compact, for any given region $C$ intersecting some sightline $\overline{a \varphi(t)}$ there exists some minimal value $t_{C}$ with $\overline{a \varphi\left(t_{C}\right)}$ intersecting $C$ before $B$. (Note that if one such line intersects $C$ before $B$, all such lines that intersect $C$ must do so before $B$, as $B$ and $C$ are convex.) Since $\overline{a \varphi(1)}$ is occluded, at least one such interloping region exists. Pick the region $C$ with $t_{C}$ minimal, so that $\overline{a \varphi(t)}$ is unobstructed for $t<t_{C}$; if more than one such region exists, pick the one closest to $a$ along the line $\overline{a \varphi\left(t_{C}\right)}$. Call the first point of obstruction $c$. Then $A$ is adjacent to $C$ via the sightline $\overline{a c}$. Furthermore, each point on $\overline{c b}$ lies on $\overline{a \varphi(t)}$ for some $t<t_{C}$ and thus must be unobstructed by the definition of $t_{C}$. Therefore, $C$ is also adjacent to $B$ via the sightline $\overline{c b}$, and so the edge $A B$ is part of the triangle $A B C$.

We now proceed to the proof of Theorem 1.

Proof of Theorem 1. Let $A$ and $B$ be mutually visible regions in a convex compact visibility representation of $G$. Because $A$ and $B$ are compact convex regions, we can find common external tangent lines $\ell_{1}$ and $\ell_{2}$ to $A$ and $B$. Let $a_{i}$ (resp. $b_{i}$ ) be a point in the intersection of $A$ (resp. $B$ ) and $l_{i}$, as shown in Fig. 2. If any other region contains a point in the closed region bounded by $A, B$, and these two tangent lines, then this point blocks some sightline between $A$ and $B$. Therefore, we can apply Lemma 1 to conclude that the edge corresponding to $A B$ is part of a triangle. 


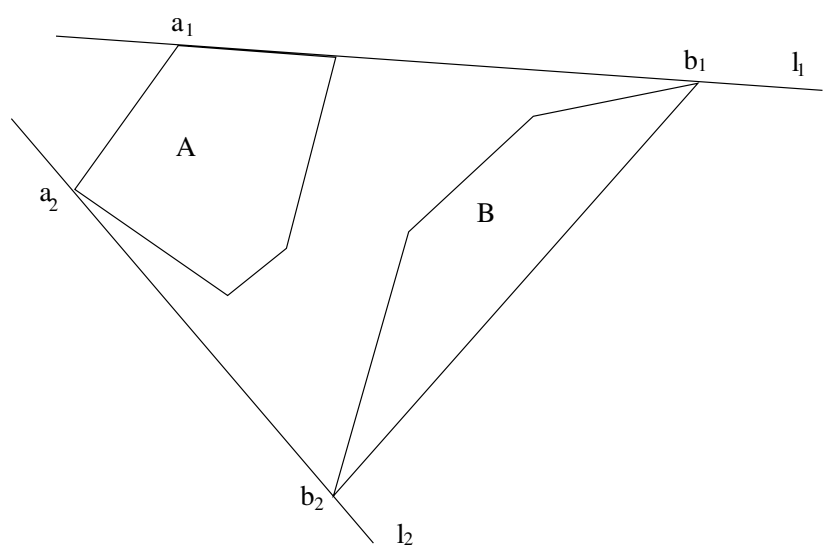

Fig. 2. Regions $A$ and $B$ with external tangent lines $l_{1}$ and $l_{2}$.

We may assume, therefore, that no such region exists. If every other region is contained either in the area bounded by $A, \ell_{1}$, and $\ell_{2}$ (and on the opposite side of $A$ from $B$ ) or that bounded by $B, \ell_{1}$, and $\ell_{2}$, it is clear that $A B$ must be a bridge. Otherwise, we have one of two cases:

Case 1: Some region has nontrivial intersection with both some $\ell_{i}$ and the open halfplane on the opposite side of that line from $A$ and $B$. Without loss of generality, we may assume that this line is $\ell_{1}$, and that the region intersects $\ell_{1}$ on the opposite side of $A$ from $B$.

Let $C$ be the first such region encountered by traveling along $\ell_{1}$ from $A$ away from $B$. Because $C$ extends at least to $\ell_{1}$, we can construct, as in Fig. 3 , a sequence of points $\left\{c_{i}\right\}$ in $C$ so that $\left\{c_{i}\right\}$ converges to $c \in C \cap \ell_{1}$, and each point $c_{i}$ is on the opposite side of $\ell_{1}$ as $A$ and $B$.

Now, let $D$ be any region besides $C$ that intersects the open half-plane on the other side of $l_{1}$ from $A$ and $B$. Then $D$ will not intersect the segment $\overline{c b_{1}}$ by our choice of $C$. Since $D$ and $\overline{c b_{1}}$ are compact sets, the distance $\delta_{D}$ between them is positive; let $\delta$ be the smallest such $\delta_{D}$. Then, if we pick $i$ large enough so that $d\left(c_{i}, c\right)<\delta$, the sightlines $\overline{c_{i} a_{1}}$ and $\overline{c_{i} b_{1}}$ must be unobstructed. Thus $A B C$ is a triangle.

Case 2: No such region exists; therefore, some region must lie entirely on the opposite side of some $\ell_{i}$ from $A$ and $B$. We may suppose without loss of generality that this line

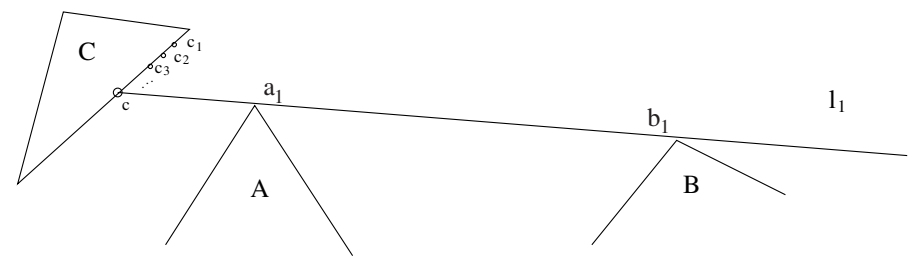

Fig. 3. A sequence of points $c_{n} \rightarrow c$. 
is $\ell_{1}$. Consider the set of all regions $D_{i}$ lying on the opposite side of $\ell_{1}$ from $A$ and $B$. Since each region is compact, and does not intersect $\ell_{1}$ itself, each has some minimum distance $\delta_{i}>0$ to $\ell_{1}$. Choose a region $D$ with minimum distance $\delta$, and let $d \in D$ be such that $d\left(d, \ell_{1}\right)=\delta$. Now, consider the sightlines $\overline{d a_{1}}$ and $\overline{d b_{1}}$. These sightlines, which lie entirely on the opposite side of $\ell_{1}$ from $A$ and $B$, must be unobstructed, for any intervening region $E$ would necessarily have $d\left(E, \ell_{1}\right)<\delta$. Therefore $D A B$ is a triangle.

Thus, in both cases, the edge $A B$ is part of a triangle. This completes the proof of the theorem.

The basic concept used in the proof of Theorem 1 is the division of $\mathbb{R}^{2}$ into three areas: the convex hull of $A \cup B$, the area blocked from $A$ by $B$ or from $B$ by $A$, and the remainder. Using this division, we showed that if any region $C$ intersects either the first area or the third, then $A B$ is part of a triangle, and if no such region exists, then $A B$ is a bridge. This division and the proof carry through in the case where the representing regions are not required to be convex. The details are similar to those of the proof of Theorem 1 , though slightly more technical, and are left to the reader.

Theorem 2. If $G$ is a compact visibility graph, then every edge of $G$ is either a bridge or part of a $K_{3}$.

In light of this result, one might wonder whether or not the property of convexity is irrelevant to the set of representable graphs; that is to say, whether all compact visibility graphs are compact convex visibility graphs. However, Fig. 4 shows an example of a graph that is a compact visibility graph, but not a convex compact visibility graph.

One might also wonder whether the converse of Theorem 2 is true, that is, whether or not every graph satisfying the conclusion of Theorem 2 is a compact visibility graph. This is easily shown to be false, however. To construct a counterexample, we make use of the following lemma.

Lemma 2. Suppose that $G$ is a visibility graph of any type, and $v$ is a vertex all of whose neighbors are pairwise adjacent in $G$. Then $G-\{v\}$ is a visibility graph of the
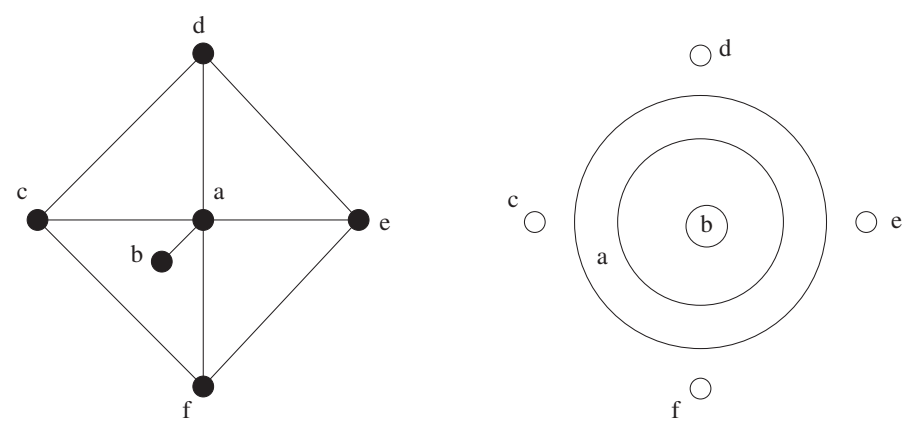

Fig. 4. A graph $G$ which is a compact visibility graph, but not a convex compact visibility graph, with a representation as the former. Any representation of $G$ as a convex compact visibility graph must have the wheel essentially as shown, which leaves no possible position for the pendant vertex. 


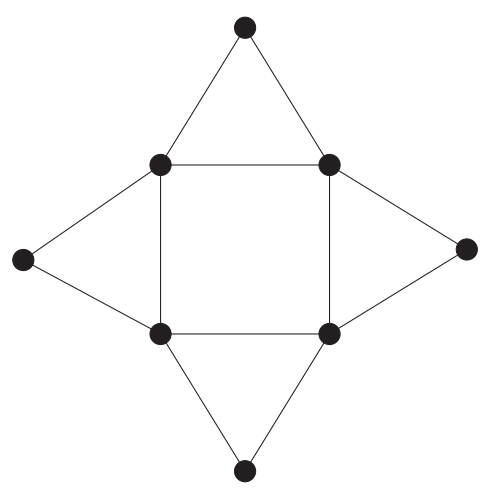

Fig. 5. A counterexample to the converse of Theorem 2.

same type. (By type of a visibility graph, we mean a type of restriction on the allowable regions in its representation.)

Proof. Construct a visibility representation of $G$, and remove the region corresponding to $v$. Consider any edge $A B$ in the resulting visibility graph, with corresponding sightline $\overline{a b}$. If we consider $\overline{a b}$ in the original representation, the only region that can possibly block it is the region corresponding to $v$. However, if this is the case, then $A$ and $B$ are each connected to $v$ using subsegments of $\overline{a b}$ as sightlines, so, by the hypothesis, $A$ and $B$ are adjacent in the original graph. Furthermore, if $v$ does not block $\overline{a b}$, then $A$ and $B$ are certainly adjacent in the original graph. Thus the edge $A B$ also occurs in $G-\{v\}$. Clearly, $G-\{v\}$ is also a subgraph of the resulting visibility graph, so the two are identical, and the lemma is proven.

Now, consider the graph $G$ shown in Fig. 5, which satisfies the conclusion of Theorem 2. Suppose $G$ were a compact visibility graph; by Lemma 2, we could then remove the four outside vertices to obtain a representation of $C_{4}$ using compact regions. However, no such representation exists (as $C_{4}$ does not satisfy the conclusion of Theorem 2), and so $G$ cannot be a compact visibility graph.

In the proof of Lemma 2 we made explicit use of the geometry of the visibility representation of $G$. Indeed, the geometry of this representation yields a condition on the graph itself, as shown in the following theorem.

Theorem 3. Suppose that there exists a convex compact visibility representation of $G$ such that two sightlines $\overline{a b}$ and $\overline{v w}$ intersect, with $a, b, v, w$ contained in distinct regions $A, B, V, W$. Then the vertices $A$ and $B$ are part of a $K_{4}$ inside $G$.

Proof. If the regions $A$ and $B$ are both contained in the line $\overleftrightarrow{a b}$, or if either region is a point, the proof is fairly straightforward and is left to the reader. Suppose not; then, without loss of generality, there exists some point $a_{0} \in A$ not on $\overleftrightarrow{a b}$.

We define a point $a^{\prime}=(1-\alpha) a+\alpha a_{0}$, where $\alpha<1$ is sufficiently small that $\overline{v w}$ still crosses $\overline{a^{\prime} b}$ and $\overline{a^{\prime} b}$ is still a sightline. (If this latter condition were impossible, we 
could construct a sequence of points in some other region converging to $\overline{a b}$, and thus $\overline{a b}$ would also not be a sightline.) We furthermore require that $B$ not be contained in the line through $a^{\prime}$ and $b$.

Now, we have $\overleftrightarrow{a^{\prime} b}$ not tangent to $A$, and thus for $b^{\prime}$ sufficiently close to $b$ we have $\overleftrightarrow{a^{\prime} b^{\prime}}$ not tangent to $A$. Pick $b_{0} \in B$ not on the line $\overleftrightarrow{a^{\prime} b}$. Then take $b^{\prime}=(1-\beta) b+\beta b_{0}$ where $\beta$ is sufficiently small that $\overline{a^{\prime} b^{\prime}}$ is a sightline and crosses $\overline{v w}$, and so that the line $\overleftrightarrow{a^{\prime} b^{\prime}}$ is not tangent to $A$. Then $\overleftrightarrow{a^{\prime} b^{\prime}}$ is tangent to neither $A$ nor $B$, for it has points from both regions on either side of it.

Because of this construction, we can assume without loss of generality that our extended sightline $\overleftrightarrow{a b}$ is tangent to neither $A$ nor $B$

Let $\delta>0$ be the minimum distance between any pair of regions; these distances are well defined, as all regions are compact. For any line segment $\overline{j k}$, let $f(\overline{j k})$ be the length of the projection of $\overline{j k}$ onto a line perpendicular to $\overline{a b}$, and let $g(\overline{j k})$ be the length of the projection of $\overline{j k}$ onto a line parallel to $\overline{a b}$; then $f$ and $g$ are always non-negative. Let $x=\inf \{f(\overline{j k})\}$, where the infimum is taken over all sightlines $\overline{j k}$ (between regions besides $A$ and $B$ ) intersecting $\overline{a b}$. It is a simple consequence of compactness and the non-tangency of $\overleftrightarrow{a b}$ to $A$ and $B$ and compactness that we have $x>0$.

Now, since the regions are compact and the line $\overleftrightarrow{a b}$ is tangent to neither $A$ nor $B$ we can pick $\theta$ sufficiently small so that a line drawn from $a$ (resp. $b$ ) at an angle at most $\theta$ from $\overline{a b}$ must intersect $B$ (resp. A) before any other region. Pick $\varepsilon>0$ so that $\varepsilon<\delta \sin \theta$. Let $\overline{c d}$ be a sightline crossing $\overline{a b}$ with $f(\overline{c d})<x+\varepsilon$, and let $C$ and $D$ be the regions containing $c$ and $d$, respectively. We claim that $A B C D$ is a $K_{4}$ in $G$. To verify this claim, we establish the existence of edges $C A, C B, D A$, and $D B$.

Let $K$ be the triangle $c a d$ in the plane, and consider the visibility graph $H$ defined by the regions $\{X \cap K\}$, where $X$ ranges over all the regions intersecting $K$ from our original visibility representation of $G$; see Fig. 6 . As $K$ is convex, and all of these new regions are contained in $K$, all relevant sightlines will also be contained in $K$; furthermore, $H$ is clearly a subgraph of $G$. Now, $H$ is a visibility graph, and $C D$ is an edge of $H$. By Theorem 1, we know that $C D$ is either a bridge or part of a triangle.

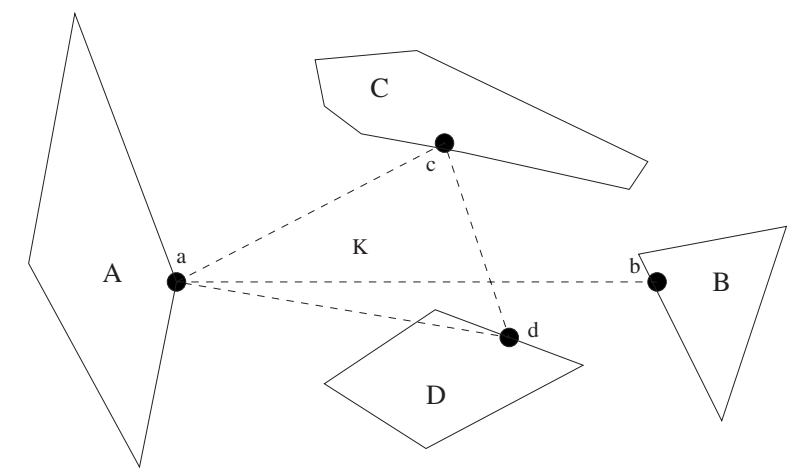

Fig. 6. Triangle $K=c a d$. There may be other regions along the edges $c a$ and $d a$, but we will show that $C$ and $A$ are mutually visible, as are $D$ and $A$. 
Since $c$ and $d$ are on opposite sides of the unobstructed sightline $\overline{a b}$, no region besides $A$ can intersect both $\overline{c a}$ and $\overline{a d}$. Taking the sequence of regions encountered along $\overline{c a}$ gives a path from $C$ to $A$ in the graph $H$, and similarly taking the sequence of regions along $\overrightarrow{a d}$ gives a path from $A$ to $D$. Composing these yields a path from $C$ to $D$ not including the edge $C D$, since $D$ cannot appear in the first part (as it appears in the second), and $C$ cannot appear in the second. Consequently, removing the edge $C D$ cannot disconnect the graph $H$, so $C D$ cannot be a bridge in $H$.

Therefore, $C D$ must be part of a triangle in $H$, so there must exist some region $E$ with both $C E$ and $D E$ edges of $H$. Unless $E=A, E$ must lie either entirely above the unobstructed sightline $\overline{a b} \cap K$ or entirely below it. However, if $E$ lies above this line, the sightline $\overline{d^{\prime} e}$ corresponding to the edge $D E$ crosses $\overline{a b}$. Furthermore, as $e \in K$ and the distance from $e$ to $c$ is at least $\delta$, it follows from our choice of $\varepsilon$ that $f\left(\overline{d^{\prime} e}\right)<x$, a contradiction. Similarly, $E$ cannot lie below $\overline{a b} \cap K$, as then $f\left(\overline{c^{\prime} e}\right)<x$. Consequently, $E$ must be the region $A$, so that both of the edges $C A$ and $D A$ occur in $H$ and, therefore, in $G$. Interchanging $A$ and $B$ shows that the edges $C B$ and $D B$ must also occur in $G$, so that $A B C D$ forms a $K_{4}$ as desired.

Theorem 3 rules out an additional class of graphs, and places constraints on possible representations of many more graphs. Noting that if a visibility representation has no crossing sightlines, the corresponding graph is planar, we obtain the following corollary.

Corollary 1. Every convex compact visibility graph either is planar or contains a $K_{4}$.

An example of a graph that this corollary excludes from being a convex compact visibility graph is shown in Fig. 7.

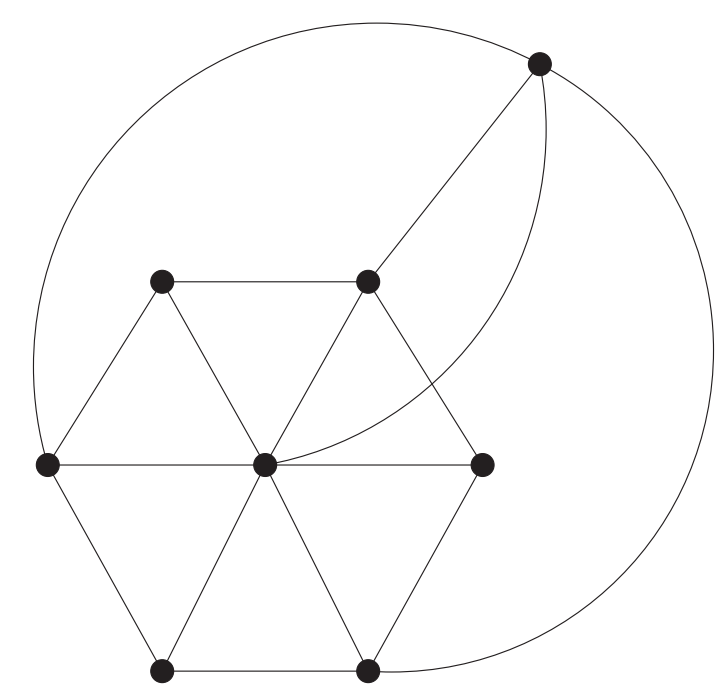

Fig. 7. This graph neither is planar nor contains a $K_{4}$, and thus cannot be a convex compact visibility graph. 


\section{Examples of Compact Visibility Graphs}

Theorem 2 shows that many simple graphs are not compact visibility graphs; in particular, $C_{n}$ is not a compact visibility graph for $n>3$. Furthermore, it is easy to check that every nonempty compact visibility graph must be connected. Nevertheless, we can construct large families of compact visibility graphs.

Theorem 4. If $G$ has a plane drawing such that all of the internal faces are triangles, then $G$ is a compact visibility graph.

Proof. Assume first that $G$ has no cut vertices, that is, vertices $v$ such that $G-\{v\}$ is disconnected. We start with a plane drawing of $G$, which we may assume has convex boundary face, and construct from this drawing a compact visibility representation of $G$. Consider any edge $v w$. We break $v w$ at an arbitrary point in its interior, and interlace the halves of $v w$ together at this point, as shown in Fig. 8. We then take as the representing region for $v$ the union of all the half-edges containing $v$. It is clear that every edge $v w$ corresponds to a sightline; furthermore, every sightline lies entirely in the interior of some face of the drawing, and thus only connects regions corresponding to the vertices of this face. As these vertices form a $K_{3}$ in the graph, there are no extraneous edges added, and so this assignment of regions shows that $G$ is in fact representable as a visibility graph.

In the case where $G$ has a cut vertex $v$, we can split the vertex as in Fig. 9 and then represent the resulting graph as a visibility graph as before, except that we do not do the above for the edge $v_{1} v_{2}$. This yields a representation of $G$ as a visibility graph.

A simple corollary of Theorem 4 , as every tree has a plane drawing with no internal faces, is the following.

Corollary 2. All trees can be represented as compact visibility graphs.

In fact, all trees are convex compact visibility graphs, or even compact disk visibility graphs.
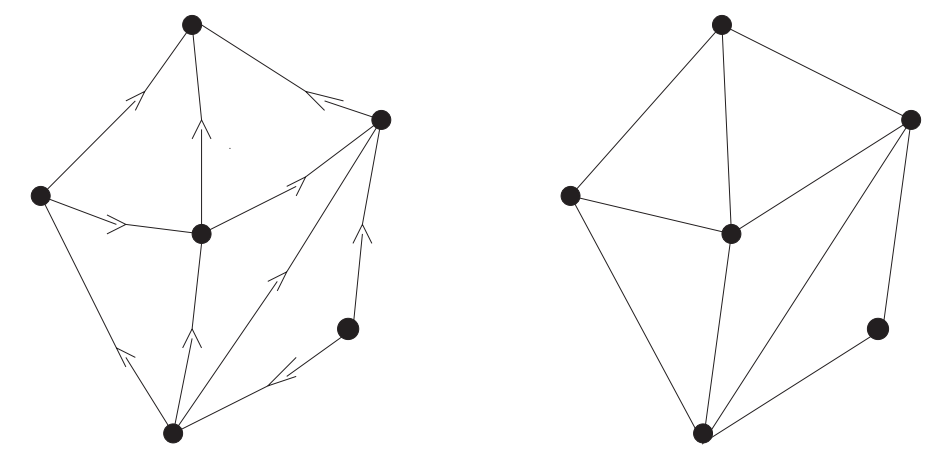

Fig. 8. An example of the construction described in Theorem 4. The graph represented is shown on the right, and its representation as a compact visibility graph on the left. 


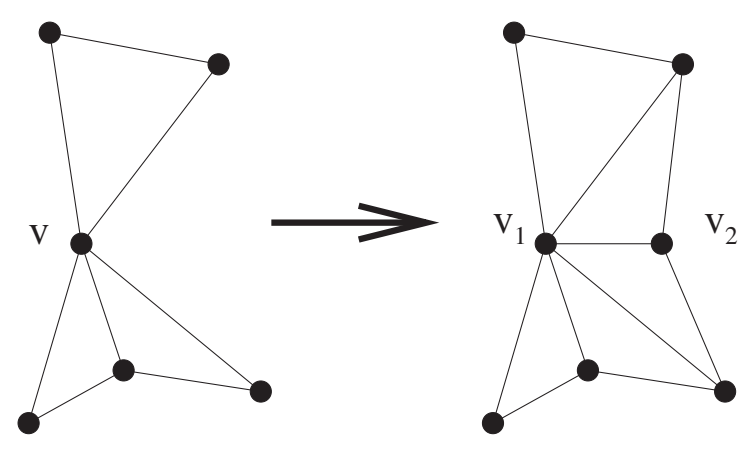

Fig. 9. Elimination of cut vertices.

Since $C_{n}$ is not a compact visibility graph for $n>3$, one might suspect that a planar graph $G$ is a visibility graph if and only if it has a plane drawing such that all of the internal faces are triangles. This, however, turns out to be false. Consider the case of $K_{1,1, n}$. This is easily seen to be a compact visibility graph (as in Fig. 10), but it is a planar graph with no such drawing for $n \geq 3$. The key point here is that the induced subgraph on the four-sided face is not $C_{4}$; given this, we conjecture that the following modification of the converse holds.

Conjecture 1. A planar graph $G$ is a compact visibility graph if and only if it has a plane drawing such that for all internal faces $F$ of $G$, the subgraph $G_{F}$ induced by the vertices of $F$ is a compact visibility graph.

\section{The Noncompact Case}

Throughout the previous two sections of this paper, we have assumed that our representing regions are compact. In this section we give our rationale for this assumption, as well as some comments about the noncompact theory.
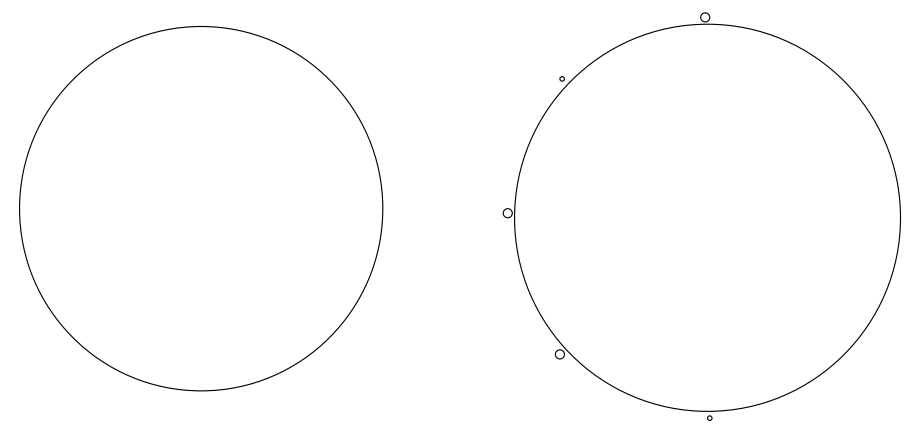

Fig. 10. Representation of $K_{1,1, n}$ as a convex compact visiblity graph. The two large circles correspond to the 1-element partite sets; the other $n$ vertices are sufficiently small and close to the large circle that no two can see each other. 

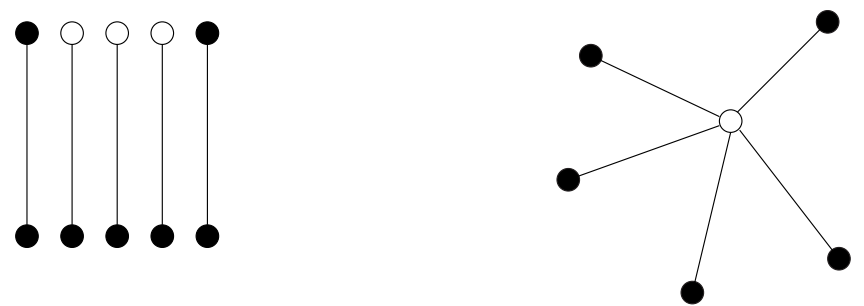

Fig. 11. Two representations of $C_{n}$ as a visibility graph using connected, non-compact regions. In the first representation, we have $n$ parallel segments, all open on both ends except the first and last, which are closed at one end. In the second representation, we have $n$ half-open line segments of equal length, no two diametrically opposed, emanating from a single point.

Theorem 2 does not hold in the noncompact case. Indeed, the cycles $C_{n}$ are visibility graphs, as shown by the two representations in Fig. 11.

Furthermore, if we eliminate the hypothesis of compactness, we can even realize disconnected graphs as visibility graphs. Let $A$ be the set $\{(x, y) \mid 0<x \leq 1, y=$ $\sqrt{x} \sin (1 / x)\}$, let $B$ be the set $\{(x, x+y) \mid(x, y) \in A\}$, and let $C$ be the single point $(0,0)$, as represented in Fig. 12. Then the only unblocked sightlines are between $A$ and $B$, and so $\{A, B, C\}$ is a visibility representation of a disconnected graph.

Combining the technique of interlacing presented in Fig. 12 with the second method for realizing $C_{n}$ presented in Fig. 11, we can realize any connected planar graph as a visibility graph. Given a face $F$, for each vertex $v$ we construct in the interior of $F$ interlocking spirals $s_{v, F}$, each having infinitely many turns; one of these spirals is shown in Fig. 13. The region corresponding to $v$ then consists of the union over all faces $F$ containing $v$ of the spirals $s_{v, F}$.

While it is not true that we can represent any graph as a visibility graph (consider the case of two isolated vertices), the fact that all connected planar graphs can be realized as visibility graphs leads to the following conjecture:

Conjecture 2. Any connected graph is representable as a visibility graph.

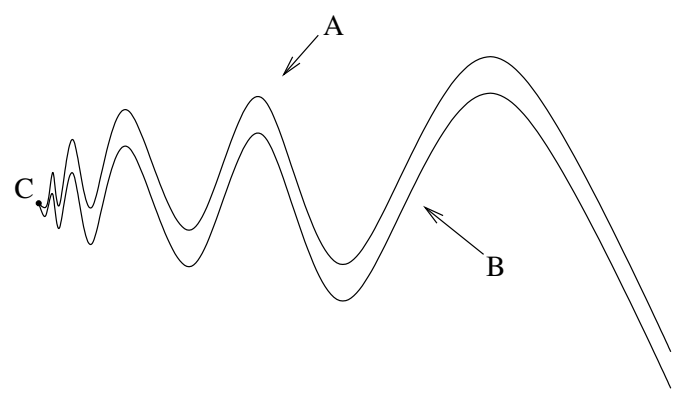

Fig. 12. A disconnected visiblity graph. Vertex $C$ can see neither $A$ nor $B$, each of which has infinitely many undulations. 


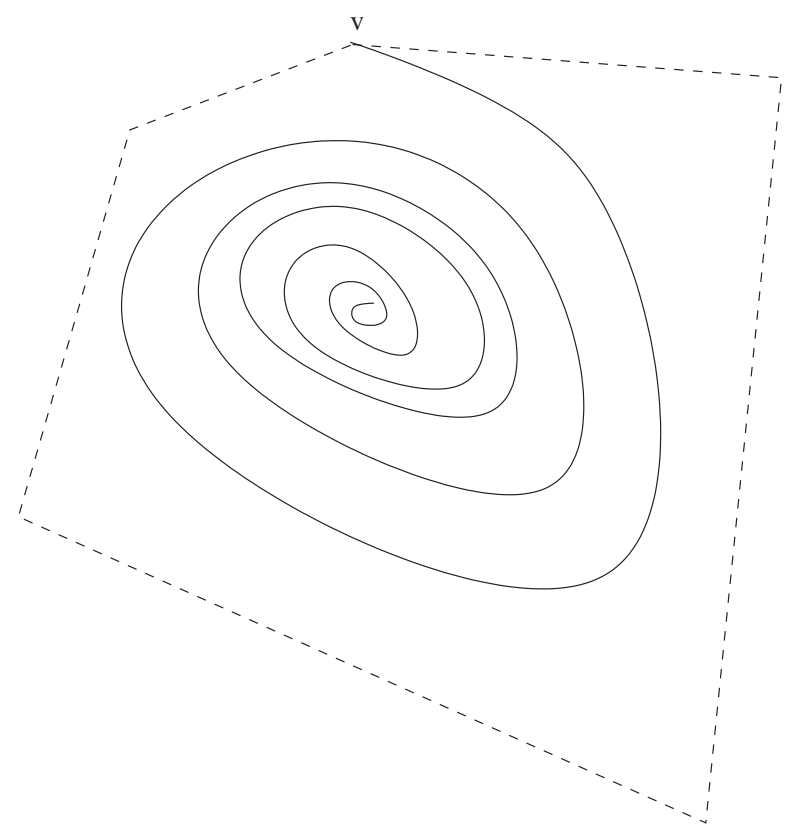

Fig. 13. Method of construction of all planar graphs as visibility graphs. The spiral shown has infinitely many turns, and is the part of the region representing $v$ corresponding to the indicated face. The edges of the face are drawn for clarity and do not appear in the visibility representation.

\section{Settings Other than $\mathbb{R}^{2}$}

It is natural to extend the concept of visibility graphs to higher-dimensional Euclidean spaces. Specifically, one might consider the case of $\mathbb{R}^{3}$. Analogues to rectangle-visibility graphs exist in $\mathbb{R}^{3}$; in particular, Fekete and Meijer [6] consider the question of which complete graphs are representable as box-visibility graphs. Alternatively, one can require that the sightlines be strictly vertical, and that each representing set lies in a horizontal plane; the question of such representations has been considered by Alt et al. [3].

In $\mathbb{R}^{3}$, Conjecture 2 is true (using our notion of visibility). Let $T$ be a spanning tree for an arbitrary connected graph $G$; using the construction shown in Fig. 1 with spheres instead of circles, we first construct a visibility representation of $T$. Then, to add an edge between two vertices $V$ and $W$, we pick any points $v \in V$ and $w \in W$, and remove all points on the interior of the line segment $\overline{v w}$. It is clear that this will only add the single sightline from $V$ to $W$, as the line has thickness 0 and all representing regions have positive thickness. Unlike in the two-dimensional case, this construction does not disconnect any regions. After all remaining edges of $G$ have been added in this fashion, one is left with a visibility representation of $G$.

In the compact case we have results similar to those in $\mathbb{R}^{2}$. With only slight modifications, the proof of Theorem 2 carries over into $\mathbb{R}^{n}$, so that Theorem 2 is true in general Euclidean spaces. This suggests the following two questions. 
Question 1. Is it true that all graphs representable as compact visibility graphs in $\mathbb{R}^{3}$ are also representable as compact visibility graphs in $\mathbb{R}^{2}$ ?

Question 2. Does there exist a positive integer $n$ such that all graphs representable as compact visibility graphs in any Euclidean space are representable as compact visibility graphs in $\mathbb{R}^{n}$ ?

Two other venues to which visibility graphs generalize easily are the torus $T^{2}$ and the sphere $S^{2}$; Mohar and Rosenstiehl [7] briefly considered the former case with restricted sightlines. In order to make sense of the general concept, we must define the notion of a straight line in both settings. We define straight lines on the torus by considering it as the quotient space $\mathbb{R}^{2} / \mathbb{Z}^{2}$; on the sphere, we take the straight lines to be the ordinary geodesics. Which graphs are representable as visibility graphs in these new settings?

\section{Conclusion}

The generalized concept of a visibility graph lends itself to various applications. Since the set of representing regions contains all of the information about the graph itself, it can be used as an encoding of information about the $n$ vertices of a graph $G$ and the $\left(\begin{array}{l}n \\ 2\end{array}\right)$ connectedness relations into a set of $n$ objects. If we require the representing regions to be disks, we have this information using $3 n$ coordinates: one triple $(x, y, r)$ for each disk.

Visibility graphs also can be used naturally to represent various kinds of networks. For instance, consider a network of army bases on a battlefield that communicate with each other via torch signals (or, if you will, lasers). The graph of this network will then be the visibility graph represented by the layout of the bases. Similarly, on a more global scale, one may wish to manipulate the situation so that one can communicate in this fashion with one's allies and at the same time intercept lines of communication between one's enemies. In this latter case of two rival alliances, it is appropriate to partition the vertices into two classes, and only consider sightlines between two regions of the same class; what pairs of networks can be realized in this fashion? The concept of visibility graphs provides a natural representation of graphs in $\mathbb{R}^{2}$ that can be used to examine these questions.

\section{Acknowledgments}

The authors thank Alice Dean for presenting the original talk which spurred the work presented in this paper, and Joseph Gallian for helpful comments on a preliminary version of it; they also thank the referees for their suggestions. 


\section{References}

1. J. Abello, H. Lin, and S. Pisupati, On visibility graphs of simple polygons, Congr. Numer. 90 (1992), 119-128.

2. P. K. Agarwal, N. Alon, B. Aronov, and S. Suri, Can visibility graphs be represented compactly?, Discrete Comput. Geom. 12 (1994), 347-365.

3. H. Alt, M. Godau, and S. Whitesides, Universal 3-dimensional visibility representations for graphs, Comput. Geom. 9 (1998), 111-125.

4. A. Dean and J. Hutchinson, Rectangle-visibility representations of bipartite graphs, Discrete Appl. Math. 75 (1997), 9-25.

5. A. Dean and J. Hutchinson, Rectangle-visibility layouts of unions and products of trees, J. Graph Algorithms Appl. 2 (1998), 21 pp. (electronic)

6. S. Fekete and H. Meijer, Rectangle and box visibility graphs in 3-D, Internat J. Comput. Geom. Appl. 9 (1999), 1-27.

7. B. Mohar and P. Rosenstiehl, Tesselation and visibility representations of maps on the torus, Discrete Comput. Geom. 19 (1998), 249-264.

8. J. O'Rourke and J. Rippel, Two segment classes with Hamiltonian visibility graphs, Comput. Geom. 4 (1994), 209-218.

9. G. Srinivasaraghavan and A. Mukhopadhyay, A new necessary condition for the vertex visibility graphs of simple polygons, Discrete Comput. Geom. 12 (1994), 65-82.

Received August 2, 2000, and in revised form April 1, 2002. Online publication March 28, 2003. 